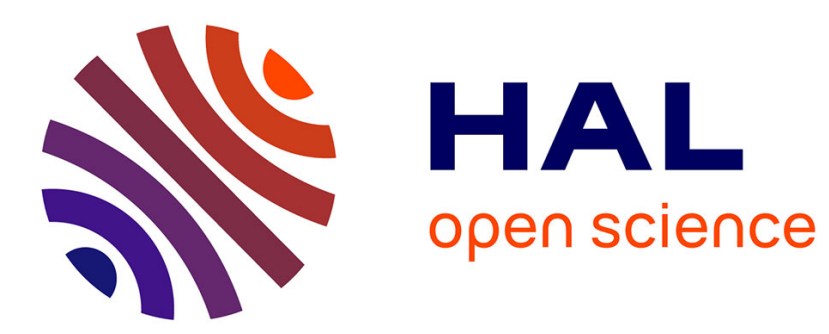

\title{
Espace de Caractérisation du Stylo Numérique
}

\author{
Sylvain Malacria, Eric Lecolinet
}

\section{To cite this version:}

Sylvain Malacria, Eric Lecolinet. Espace de Caractérisation du Stylo Numérique. Proceedings of the AFIHM Conférence Francophone sur l'interaction Homme-Machine (IHM 2008), Sep 2008, Metz, France. pp.177, 10.1145/1512714.1512749 . hal-02871296

\section{HAL Id: hal-02871296 https://hal.inria.fr/hal-02871296}

Submitted on 17 Jun 2020

HAL is a multi-disciplinary open access archive for the deposit and dissemination of scientific research documents, whether they are published or not. The documents may come from teaching and research institutions in France or abroad, or from public or private research centers.
L'archive ouverte pluridisciplinaire HAL, est destinée au dépôt et à la diffusion de documents scientifiques de niveau recherche, publiés ou non, émanant des établissements d'enseignement et de recherche français ou étrangers, des laboratoires publics ou privés. 


\section{Espace de Caractérisation du Stylo Numérique}

\author{
Sylvain Malacria \\ Telecom ParisTech \\ 46, rue de Barrault \\ 75013, Paris, France \\ malacria@telecom-paristech.fr
}

\author{
Eric Lecolinet \\ Telecom ParisTech \\ 46, rue de Barrault \\ 75013, Paris, France \\ lecolinet@telecom-paristech.fr
}

\begin{abstract}
RESUME
Malgré l'omniprésence des ordinateurs et autres PDAs, le couple papier/stylo conserve de nombreux avantages et reste largement utilisé. Son appartenance au «monde physique » a cependant pour inconvénient d'entrainer une séparation presque totale avec les applications du "monde numérique ». Dans cet article nous nous intéressons au stylo numérique et aux passerelles que ce type de technologie permet d'établir entre les objets tangibles et numériques. Nous proposons un espace de caractérisation qui est articulé autour des propriétés du stylo numérique, du papier interactif et des interactions de l'utilisateur avec ces outils.
\end{abstract}

MOTS CLES : Techniques d'interaction, Interfaces tangibles, Stylo numérique, Papier Interactif.

\begin{abstract}
Despite computers and PDAs are now so common, the pen / paper couple is still widely used in practice. However, as it belongs to the "physical world", it is strongly separated from "digital world" applications. In this paper, we focus on digital pen technology and explore the bridges it can provide between tangible and digital objects. We propose a framework that relies on the properties of digital pen and interactive paper and on how users can interact with these tools.
\end{abstract}

CATEGORIES AND SUBJECT DESCRIPTORS: H5.2 [Information interfaces and presentation]: User Interfaces.

GENERAL TERMS: Design, Human Factors.

KEYWORDS: Interaction Techniques, Tangible User Interfaces, Digital Pen, Interactive Paper.

\section{INTRODUCTION}

Malgré 1'omniprésence des dispositifs numériques la consommation de papier ne cesse d'augmenter [26] et le travail de bureau en dépend encore largement. Le papier comporte l'intérêt d'être léger, malléable et peu coûteux tandis que les outils numériques facilitent la diffusion, la duplication et l'indexation de l'information. De fait, dans leurs usages actuels, le papier et les dispositifs informatiques sont davantage complémentaires que concurrents. Ils appartiennent cependant à deux mondes fortement séparés : les objets physiques d'une part et les représentations numériques d'autre part. Dans cet article nous nous intéressons au couple stylo numérique / papier « interactif » et aux passerelles que ce type de technologie peut permettre d'établir entre objets tangibles et numériques.

Dans les sections suivantes, nous présentons un espace de caractérisation des techniques d'interaction du couple stylo numérique / papier interactif. Cet espace permet d'identifier les tendances et lacunes actuelles en ce qui concerne les outils utilisés et les usages associés. Vient ensuite la synthèse de cet espace qui introduit des perspectives.

\section{DEFINITION DE L'ESPACE DE CARACTERISATION}

Le stylo numérique ne semble pas pour l'instant avoir fait l'objet d'une définition formelle. Nous proposons donc la définition suivante: un stylo numérique est d'abord un stylo «classique » (c'est-à-dire, d'après Wikipedia [37], un outil de forme allongée pour faciliter sa préhension, qui sert le plus couramment à écrire ou dessiner et qui dispose d'un réservoir d'encre), «augmenté » de composants numériques (au minimum d'un capteur, une batterie et une mémoire). Le dispositif de capture du tracé doit permettre d'interpréter de manière précise les mouvements de la pointe du stylo sur le papier. Le tracé manuscrit doit pouvoir être mémorisé de façon autonome par le stylo (c'est à dire en l'absence d'un ordinateur), puis être transféré ultérieurement sous forme numérique à un dispositif informatique (ordinateur, PDA, téléphone portable, etc.) qui sera en mesure de les interpréter de manière adéquate.

Un stylo numérique diffère donc d'un stylet de tablette graphique par son autonomie et sa capacité d'être utilisé 
sans PC. De plus, comme un stylo standard, il dépose de l'encre sur une feuille contrairement à des dispositifs tels que Scrivo.1 [44], ioTracker [25] ou [7]. Enfin, il est intéressant de signaler que l'autonomie du stylo numérique, qui est bien supérieure à celle d'un PDA ou d'un ordinateur portable, en fait un outil particulièrement approprié pour une utilisation en situation de mobilité.

L'accomplissement d'une tâche utilisant le stylo numérique passe par une séquence d'interaction de l'utilisateur (figure 1). Il écrit avec le stylo, éventuellement en situation de mobilité, puis il transfère les tracés, de manière continue ou différée, à un système informatique (ordinateur, PDA, etc.) qui les interprète. Le système peut-être équipé de dispositifs annexes tels qu'une imprimante ou un vidéoprojecteur.

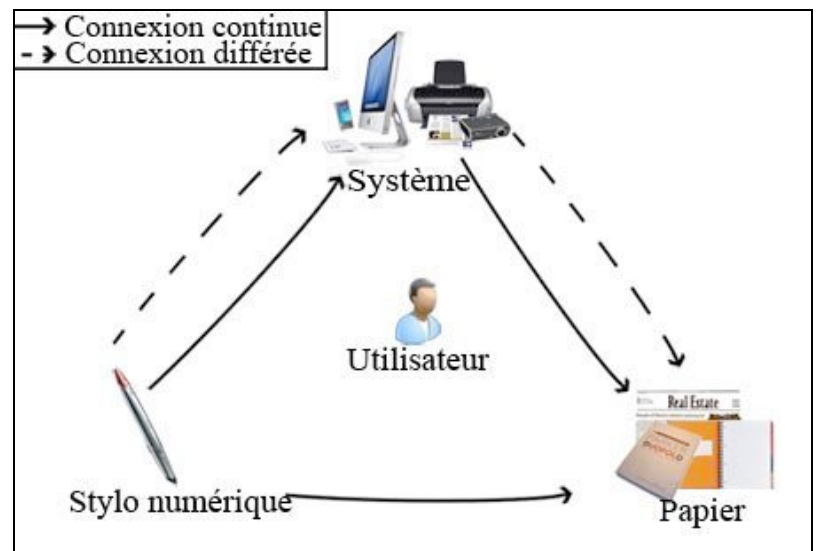

Figure 1 : Représentation de la communication interdispositifs (Système, Stylo numérique et Papier)

Les axes de l'espace de caractérisation que nous proposons s'appuient sur trois acteurs majeurs : le stylo, le papier et l'utilisateur. Par la suite, nous détaillons les propriétés de chacun de ces axes en nous appuyant sur des exemples de l'état de l'art.

\section{PROPRIETES DU STYLO NUMERIQUE}

Dans cette section, nous caractérisons les propriétés techniques des stylos numériques : système de localisation, capacité à associer un identifiant temporel et mode de communication avec le système.

\section{Système de localisation}

La principale différence entre les technologies existantes est la dépendance par rapport au type de papier. Celui-ci peut être quelconque, spécifique, ou nécessitant une préparation appropriée.

La localisation par triangulation d'ondes acoustiques est typique du premier cas. Elle est par exemple utilisée par le stylo Epos [39] qui localise la position du stylo par rapport à un capteur acoustique fixé en haut de la page au moyen d'un clip que l'utilisateur doit ajuster. Le stylo émet des ondes lorsqu'il écrit ce qui permet au capteur de déterminer sa position sur la page. Cette technologie a pour avantage d'être compatible avec du papier ordinaire mais nécessite, chaque fois que l'on change de page, de devoir refixer le capteur (qui ressemble à une sorte de clé USB) en haut de celle-ci.

A contrario, la technologie Anoto [36,40,41,42], qui requiert du papier micro-tramé, est un bon représentant du second cas de figure. Le stylo est équipé d'une mini caméra infrarouge qui détermine sa position sur la page en détectant où il se trouve par rapport à la trame. Cette trame est généralement pré-imprimée par le fournisseur du papier mais peut être rajoutée sur du papier normal à condition de disposer d'une imprimante ayant la résolution adéquate (1000 DPI minimum) et d'utiliser de l'encre appropriée (à base de noir de carbone).

Le type de localisation offert par cette technologie peut être qualifié de «quasi-absolu» dans la mesure où il permet non seulement d'enregistrer le tracé mais aussi d'identifier la page physique correspondant à chaque point du tracé. Le codage Anoto permet en effet de définir une trame d'une taille considérable (surface équivalente à près de 60 millions de $\mathrm{km}^{2}$ ). Chaque page peut ainsi être associée à une partie différente de la trame. Ceci permet par simple lecture optique du stylo à un point quelconque d'identifier à la fois la page et la position relativement à cette page. Toutefois, pour des raisons pratiques et commerciales, des pages différentes sont souvent associées à une même partie de trame. C'est par exemple le cas des cahiers Oxford EasyBook M3 [43] où un modèle commercial de cahier utilise une trame donnée (toutes les «instances » de ce modèle de cahier utilisent donc la même trame). La localisation est alors absolue par rapport à un modèle de cahier : des tracés effectués aux mêmes pages de cahiers de même modèle seront indiscernables, à moins d'une identification préalable de l'instance de cahier utilisé.

Contrairement aux techniques comme la triangulation, le codage «quasi-absolu » permet de réécrire sur une page précédemment annotée sans décalage entre les diverses couches d'écriture (dans le cas de la triangulation le capteur acoustique est rarement fixé exactement au même endroit à chaque utilisation). De plus, elle permet d'identifier avec précision n'importe quel point de la page (ou du cahier) en posant de nouveau le stylo dessus. Cette propriété est intéressante car elle permet d'établir une correspondance bi-univoque entre les mondes physique et numérique. De ce fait, il est possible d'associer des « actions » réalisées dans l'espace numérique aux localisations dans l'espace physique. Ces actions pourront être ré-effectuées ultérieurement chaque fois que l'on utilise le stylo, ceci apportant une forme d'interactivité au papier. Nous qualifierons par la suite de «marques actives » les tracés physiques auxquels sont associés des actions numériques. 


\section{Identifiant temporel}

Une autre propriété importante de certains stylos numérique est leur capacité d'associer un identifiant temporel à chaque événement (et donc à chaque point du tracé). Cette caractéristique permet non seulement de détecter des combinaisons d'événements typiques (par exemple des clics, des clics longs, des double-clics), mais aussi d'établir des correspondances temporelles entre les lieux des tracés et des événements externes survenus dans l'environnement dans lequel était utilisé le stylo ([1], [35]). Par exemple, dans le contexte d'une classe augmentée, une note de l'élève inscrite à un instant $t$ pourra être associée au fait que le professeur a au même instant affiché une certaine diapositive à l'écran. La double correspondance spatiale/temporelle permet ainsi de définir des triplets (marque active, événement environnemental, action informatique associée) où chacun de ces éléments sont en relation tri-univoque.

\section{Connexion stylo / ordinateur}

Une dernière propriété du stylo est le mode de communication stylo / ordinateur, qui peut être continu ou discontinu. Dans le premier cas le stylo est connecté à l'ordinateur et lui envoie les tracés en continu. Il peut alors être vu comme un dispositif d'interaction supplémentaire de l'ordinateur. Dans le second cas, le stylo fait office de dispositif nomade qui est épisodiquement synchronisé à l'ordinateur. Les stylos qui disposent d'un processeur embarqué et de dispositifs de retour évolués, comme [40], [41] et [42], constituent un cas particulier dans la mesure où ils peuvent être vus à la fois comme un stylo numérique et un ordinateur.

Signalons enfin que les technologies précédentes semblent avoir supplanté des techniques comme le tracking vidéo, utilisé par exemple dans DigitalDesk [32], probablement car elles nécessitaient du matériel supplémentaire (une caméra) et une mise en œuvre (phase de calibration) plus complexe. La technologie s'appuyant sur la navigation optique de Scrivo.1 [44], qui interprète les mouvements de la pointe sur une surface comme le fait une souris optique, pourrait être appliquée aux stylos numériques, mais aucun modèle commercial ne l'exploite, peut être à cause d'une précision insuffisante ou parce que cette technologie ne permet pas de connaitre la position absolue du stylo sur le papier.

\section{PROPRIETES DU PAPIER}

D'après notre définition, le stylo numérique est utilisé par interaction de sa pointe sur la surface d'une feuille de papier. Cet axe de classification s'appuie sur deux propriétés : (1) La topologie du support papier et (2) sa capacité à superposer des couches d'affichage.

\section{Topologie du papier}

Le papier se caractérise d'abord par sa forme (régulière ou irrégulière) et sa couleur. Par exemple, dans Bridging The Gap [13], Tom Hurlbutt et Scott R. Klemmer permettent à des programmeurs d'écrire sur des fiches pa- pier pour faciliter le développement de logiciels. Ces fiches peuvent avoir quatre couleurs différentes, chacune ayant sa propre signification. La transparence est une autre propriété intéressante, qui est par exemple exploitée par le papier calque. Cependant, sans doute pour des raisons techniques, cette possibilité ne semble pas avoir été déjà exploitée par les systèmes existants.

Le papier est le plus souvent disponible sous forme de blocs, livres, cahiers, classeurs, etc. comportant un ensemble de feuilles dont le regroupement fait sens. Un cahier est un outil de stockage d'information facile à transporter dans lequel on peut non seulement écrire et dessiner mais aussi rajouter d'autres documents comme des photos, des coupures, des étiquettes, etc. comme par exemple dans les cahiers augmentés de biologistes illustrés par les études [8] et [35]. Les cahiers ont aussi une dimension temporelle : ils permettent de stocker des informations sur une longue durée comme le montre le système Memento [33]. Un cahier de cours contient typiquement plusieurs chapitres d'une seule et même matière, enregistrés sur plusieurs mois. Des notes de cours pourraient également être enrichies d'informations sémantiques (préciser un titre, une définition, etc.) à l'aide d'un système de commande (un concept que nous détaillons dans la partie Interaction de l'utilisateur) comme dans [19].

A l'opposé, une feuille volante est généralement utilisée comme un consommable, un brouillon ou un support d'impression. L'impression d'un document sur des feuilles volantes est souvent employée pour faciliter la relecture et la correction. Dans [9] et [2], l'utilisateur imprime des photos sur une feuille et ajoute des annotations à l'aide du stylo. Paperproof [31], permet d'imprimer un document sur une feuille de papier et d'y apporter des corrections en annotant directement la feuille avec le stylo numérique. Après avoir imprimé un document (par exemple un document MS-Word) sur une feuille, l'utilisateur y corrige directement les fautes à l'aide de commandes gestuelles (voir la partie Interaction de l'utilisateur). Lorsqu'il connecte son stylo à l'ordinateur, les tracés physiques sont interprétés et les corrections sont appliquées au document numérique.

La taille est une autre propriété importante du papier. Une fiche papier, petit format, «autocollante » de type Post-It (utilisées dans [14]), sur laquelle l'utilisateur écrit avec le stylo numérique, peut être collée dans un livre pour l'augmenter «physiquement». Un poster est vu dans GigaPixels [34] comme un dispositif d'affichage à grande résolution et à faible taux de rafraichissement (une impression quotidienne par exemple). Couplé à un vidéoprojecteur (qui augmente alors son taux de rafraichissement), il permet dans [5] de réaliser un tableau interactif à faible coût. La taille du poster permet que plusieurs utilisateurs le consultent et interagissent avec celui-ci simultanément. 
Enfin, le papier peut être déformable. On peut par exemple lui donner une forme en trois dimensions en le pliant. Dans ModelCraft [29], Hyunyoung Song et al. exploitent cette propriété et présentent un outil d'édition de modèles 3D. L'application fonctionne comme une extension du logiciel de modélisation AutoCAD. L'utilisateur modélise une forme initiale sur l'ordinateur, par exemple un cube, dont l'impression sur papier fera office de patron. En pliant ce patron de manière adéquate, l'utilisateur de ce système dispose alors d'un équivalent physique papier de son document numérique. En dessinant sur cet objet physique à l'aide du stylo (par exemple un rond sur une des faces), la version numérique de ce cube est modifiée sur l'ordinateur, en laissant apparaitre un trou traversant le cube dans notre exemple. Enfin, le papier peut être tordu, plié ou même déchiré.

\section{Couches d'affichage}

Dans [11], Fekete et Beaudoin-Lafon présentent le modèle multi-calque d'une application graphique. S'appuyant sur ce modèle, Wendy E. Mackay et al. définissent dans The Missing Link [21] chaque page de leur cahier de note comme une feuille composée de la superposition de trois couches: (1) les informations préimprimées (comme par exemple un quadrillage), (2) les informations écrites avec le stylo et (3) des feuilles collées sur cette page. Cette décomposition étant spécifique aux cahiers nous proposons de l'étendre, dans un contexte plus général au stylo numérique, comme suit :

1) Tout d'abord, à la base du modèle, la feuille de papier en tant que telle, c'est-à-dire le substrat matériel sur lequel toutes les couches reposent.

2) D'éventuelles informations pré-imprimées viennent ensuite s'ajouter. Le contenu imprimé (par exemple une image) peut être associé à un équivalent numérique (par exemple un document JPG sur un ordinateur) comme le proposent [15] et [30]. Dans Print-NLink [24], l'impression d'une publication scientifique sur du papier associe toutes les références (sous la forme de chiffres entre crochets) aux documents numériques correspondants (PDF). En écrivant un « $\mathrm{p} »$ sur une référence à l'aide du stylo, l'utilisateur déclenche l'impression du document associé.

3) L'encre physique déposée par le stylo de l'utilisateur lors de la phase d'écriture.

4) L'encre "numérique», couche virtuelle qui correspond à l'enregistrement des tracés. Cette couche n'est pas nécessairement identique à la couche précédente. Par exemple, si on écrit sur la feuille sans encre (cartouche d'encre vide), le système pourra interpréter l'encre «numérique » alors que l'utilisateur ne verra aucune encre «physique». Plusieurs couches 3) et 4) peuvent se superposer. Cette superposition peut correspondre à plusieurs utilisateurs, plusieurs stylos ou plusieurs séquences d'écriture réparties dans le temps.
5) Viennent ensuite se superposer de manière récursive d'autres couches matérielles, comme des Post-It, des autocollants, etc. qui sont obligatoirement composées de la couche 1) et éventuellement des couches 2), 3) et 4).

6) Enfin, une dernière couche peut être présente et se superpose à toutes les autres. Il s'agit d'une couche de vidéoprojection comme dans [18], [4] ou [5] où le vidéoprojecteur superpose de l'information numérique sur la feuille de papier.

La figure 2 représente les couches de notre modèle. Chaque couche (à l'exception de la première) est optionnelle.

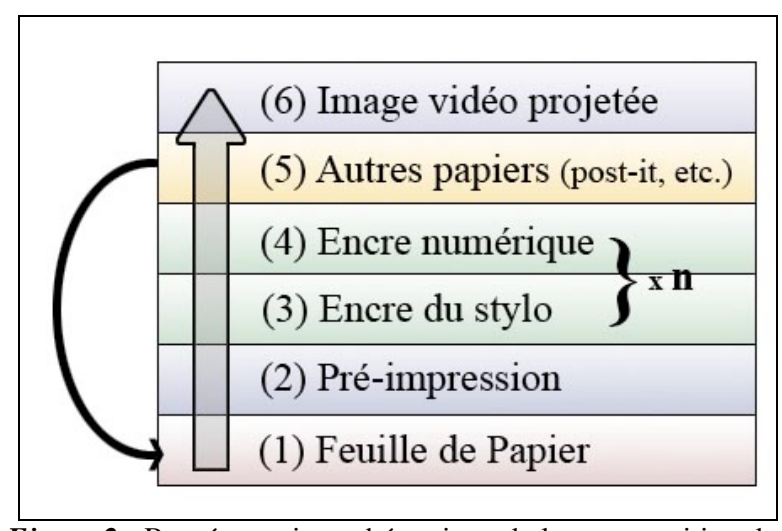

Figure 2 : Représentation schématique de la superposition des couches du papier numérique

\section{INTERACTION DE L'UTILISATEUR}

D'après Norman [23], la réalisation d'une tâche par l'utilisateur se fait selon un cycle action/perception: l'utilisateur perçoit l'état du système, l'évalue, et agit dans le but d'accomplir sa tâche. L'état du système change et entraîne un nouveau cycle. Ce cycle se base sur un ensemble varié de techniques d'interactions, en entrée comme en sortie, assimilées au terme de modalité. Nigay [22] définit cette modalité comme un couple $\langle\mathrm{D}, \mathrm{L}\rangle$, où $\mathrm{D}$ est un dispositif physique et $\mathrm{L}$ un langage d'interaction. Un dispositif physique acquiert (dispositif d'entrée) ou fournit (dispositif de sortie) une information. Un langage d'interaction est un ensemble d'expressions bien formées et significatives. Si l'utilisateur écrit sur une feuille dans le but de numériser ses tracés manuscrits, il utilise la modalité <stylo, saisie $>$.

\section{Modalités en entrée}

Les modalités en entrée du stylo concernent deux catégories classées selon les dispositifs physiques: (1) l'interaction gestuelle et (2) l'interaction audio. Ces modalités dépendent du modèle de stylo numérique et sont résumées à la figure 3 . 


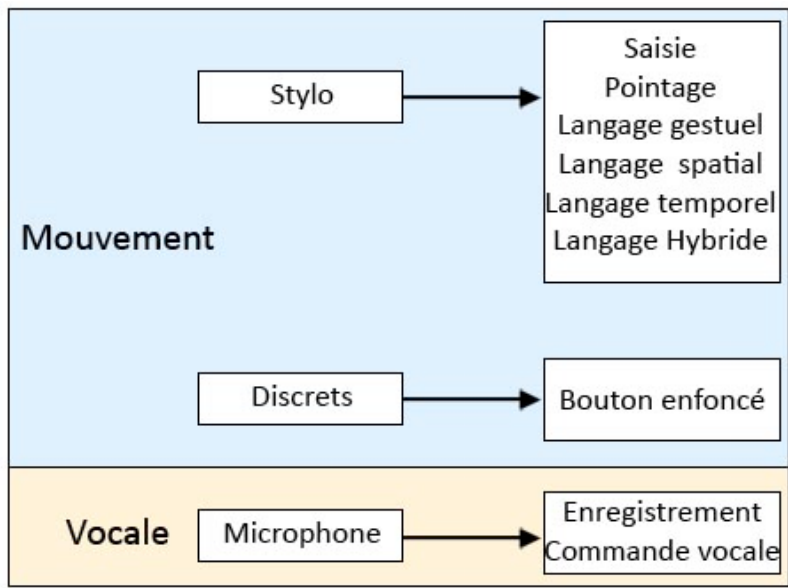

Figure 3 : Modalités en entrée

Un stylo numérique simple permet seulement d'interagir de manière gestuelle en transmettant au système des coordonnées de tracés (écriture, dessin, etc.). Ces tracés peuvent servir de saisie simple, mais connecté en continu, le stylo permet le pointage (utilisation du stylo et du papier comme une tablette graphique). Enfin, nous avons vu précédemment qu'il permet à la fois d'écrire et de réaliser diverses actions dans le monde numérique. Ceci nécessite de pouvoir facilement différencier ces deux modes. Par exemple, un utilisateur désirant envoyer un texte par email à un correspondant devra pouvoir différencier le texte (ou le tracé manuscrit de ce texte) de la commande déclenchant l'envoi. Contrairement aux stylets de tablettes graphiques, la plupart des stylos numériques actuellement disponibles ne disposent pas de boutons permettant de changer de mode. D'autres propriétés de ces dispositifs doivent donc être utilisées. Trois types de techniques (ou combinaisons de ces techniques) sont actuellement employés :

- Interaction gestuelle: certaines formes de tracés sont interprétées comme des commandes par un algorithme de reconnaissance des formes. Cette technique a priori séduisante a pour inconvénient que l'utilisateur doit apprendre un vocabulaire de gestes et être capable de les reproduire de manière non ambigüe, ce qui implique une phase d'apprentissage qui peut être rebutante. De plus, les erreurs dues au système ou à l'utilisateur peuvent compromettre l'efficacité du dispositif.

- Interaction dans des zones réservées (localisation spatiale) : par exemple, en tirant un trait vertical dans la marge pour appliquer une commande à tout un paragraphe. Ce type de technique a pour avantage d'être à la fois simple à implémenter et peu ambigu. Il a par contre pour inconvénient de nécessiter des zones spécifiques dans le document papier (perte de place), et d'avoir une expressivité limitée (peu de commandes disponibles à moins de combiner avec d'autres techniques).
- Identification temporelle : lorsqu'ils sont datés, les évènements produits par la manipulation du stylo peuvent permettre de basculer du mode saisie au mode commande, par exemple en maintenant la pointe du stylo une seconde sur le papier.

Certains stylos numériques fournissent des entrées supplémentaires comme le prototype présenté par Chunyuan Liao [20] augmenté d'un bouton qui fournit une entrée discrète matérielle supplémentaire (bouton pressé ou non).

Le Pulse Smartpen [42] dispose d'un microphone embarqué et donc d'une entrée vocale, qui pour l'instant est seulement utilisée pour l'enregistrement audio. Cette entrée pourrait aussi servir à l'exécution de commandes vocales.

\section{Modalités en sortie}

Dans [8], Costa-Cunha et Mackay mettent en avant le problème d'absence de feedback lors de l'application d'une commande en mode différé. La plupart des stylos se limitent en effet à des retours destinés à indiquer la bonne marche du matériel (LED pour indiquer que la batterie n'est pas vide, etc.). Des formes de retours supplémentaires sont parfois disponibles, soit pour permettre la confirmation des actions en mode commande, soit pour augmenter les fonctionnalités et l'autonomie par rapport au système.

Ces retours peuvent être visuels, sous forme d'écrans embarqués (comme pour [41] et [40]) ou de LEDs (comme le prototype de Chunyuan Liao [20]). Le stylo peut aussi émettre des retours audio (haut parleurs ou écouteurs). Certains modèles (Anoto notamment) permettent également un retour vibratoire, par exemple lorsque la trame n'est pas reconnue. Dans [20], un solénoïde ajouté sur le stylo offre des retours tactiles contextuels à l'utilisateur, un peu comme l'Haptic Pen [17] dans le cas des écrans tactiles. En utilisation normale, l'utilisateur garde généralement son index en contact avec le stylo. Des dispositifs de retour tactile plus sophistiqués pourraient donc être envisagés, par exemple des cellules brailles comme dans le TactiPen [16], afin de fournir des informations plus riches à l'utilisateur.

Si les retours immédiats peuvent être directement émis par le stylo, ils peuvent aussi provenir de dispositifs présents dans l'environnement lorsque le stylo y est connecté en continu. C'est par exemple le cas de [5], [6], [18], Diamond's Edge [3] ou PaperPoint [27] qui nécessitent une connexion continue à un ordinateur et un vidéoprojecteur. Néanmoins, l'utilisateur est contraint de rester à proximité de ces dispositifs. A l'opposé, dans [28] le stylo numérique est connecté en continu à un PDA qui renvoie des informations à l'utilisateur lorsqu'il pointe un lieu sur une carte touristique en papier et peut donc l'utiliser en situation de mobilité totale. C'est également le cas dans [34] où un feedback audio est émis lorsque 
l'utilisateur applique une commande à l'aide du stylo sur son cahier.

\section{SYNTHESE ET PERSPECTIVES}

En présentant de nombreuses techniques et technologies existantes ainsi qu'un espace de caractérisation dédié, les précédentes sections ont permis de mettre en évidence les principales propriétés sur lesquelles repose l'usage des stylos numériques. De plus, une table synthétique, comparant les techniques par rapport à cet espace est fournie en annexe à la référence [45].

Plusieurs remarques peuvent être faites à partir de notre étude. Tout d'abord, la quasi intégralité des travaux récents utilisent la localisation par papier micro tramé (i.e. Anoto), car cette technologie est actuellement la plus puissante et la plus simple d'usage. Par ailleurs, alors que le papier a pour caractéristique de pouvoir être utilisé sous de multiples formes (cf. sections topologie et couches), assez peu d'études exploitent cet aspect (par exemple l'utilisation de fiches mobiles, de « Post-It », de calques, etc.). Enfin, nous détaillons ci-après deux pistes d'étude qui semblent particulièrement intéressantes.

\section{Travail collaboratif}

Le couple papier/stylo numérique possède des propriétés intéressantes pour le travail collaboratif, en particulier, lorsque le stylo dispose d'un identifiant unique et est capable d'horodater les tracés. Ces caractéristiques permettent en effet au système d'identifier quel utilisateur a écrit et à quel moment. De plus, le papier est malléable et peut facilement être échangé entre plusieurs personnes. La classification d'Ellis [10] propose de classer un travail collaboratif selon deux axes (figure 4) :

- L'axe temporel décrit la manière dont travaillent les utilisateurs, qui peut être synchrone ou asynchrone.

- L'axe spatial décrit la localisation des utilisateurs au moment du travail. Ceux-ci peuvent être distants ou co-localisés (en présence l'un de l'autre dans une même pièce).

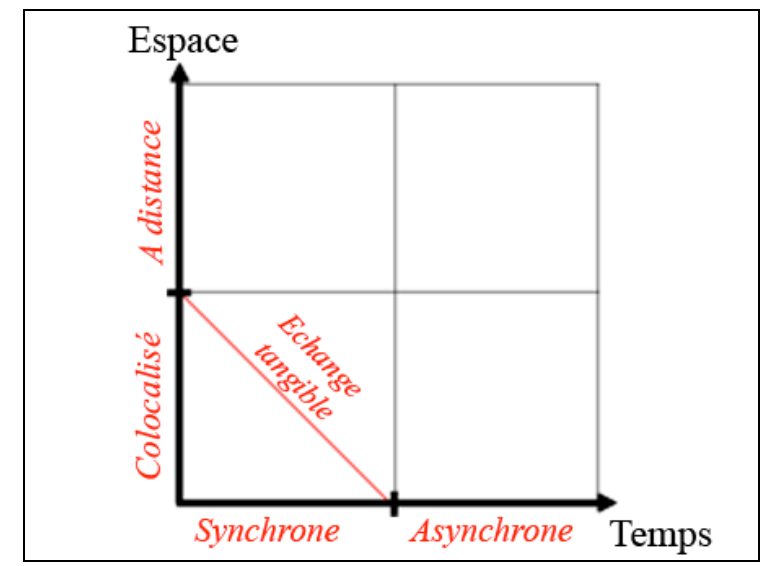

Figure 4 : Espace de classification du travail collaboratif avec le stylo numérique.
Le système PADD [12], dédié à l'édition collaborative de documents numériques, illustre le cas asynchrone à distance. Chaque utilisateur peut imprimer le document sur une feuille puis y ajouter des annotations manuscrites qui sont superposées au contenu initial du document. La nouvelle version est transmise électroniquement à tous les collaborateurs.

A contrario, la collaboration est synchrone et colocalisée avec Diamond's Edge [3]. Cet outil de dessin collaboratif permet à plusieurs utilisateurs placés autour d'une table, d'interagir avec leurs stylos et blocs notes respectifs. Les stylos sont connectés en continu à un ordinateur contrôlant un vidéoprojecteur qui projette une image sur la table et les blocs notes. Chaque utilisateur peut dessiner sur sa propre feuille (qui fait office d'espace privé) et lorsqu'il le souhaite, «glisse» son dessin sur la table (partagée par tous) à l'aide de son doigt. Son propre dessin est alors affiché dans l'espace partagé. Un autre utilisateur peut ensuite déplacer ce dessin dans son espace privé (son bloc note) et le modifier avec son stylo. Ces modifications sont alors vidéoprojetées en temps réel sur le dessin original du premier auteur.

L' « échange tangible » est un cas particulier de l'axe colocalisé synchrone qui n'est pas explicitement mis en évidence dans la classification d'Ellis et ne semble pas avoir été exploité par les techniques existantes. Le couple stylo/papier permet également aux utilisateurs de procéder à un échange matériel dans le monde physique lorsqu'ils travaillent ensemble (typiquement en échangeant temporairement une feuille de papier ou un stylo). Ce cas de figure est à distinguer d'un échange dans le monde numérique car la «physicalité » de l'action entraine des propriétés particulières. L'utilisation du stylo d'un acteur A sur le cahier d'un acteur B, peut par exemple donner des droits particuliers à $\mathrm{A}$ sur l'espace numérique de B. La matérialité du stylo et du papier permet alors une forme d'authentification, simple et efficace, qui n'a pas d'équivalent dans le monde purement numérique.

\section{Interactions avancées}

Nous avons vu précédemment que le stylo numérique n'était pas limité à la numérisation des tracés manuscrits mais pouvait également servir d'entrée à un langage de commandes. L'ajout de capteurs supplémentaires, par exemple pour détecter l'inclinaison ou la pression, permet d'envisager des techniques d'interaction plus variées et de nouveaux types d'utilisation (une mesure de pression est d'ailleurs déjà disponible sur la plupart des stylos Anoto, même si elle est pour l'instant relativement imprécise). Dans le même esprit, les stylos pourraient également être pourvus de boutons ou d'une "gomme» à l'instar de la plupart des stylets de tablettes graphiques. 
La miniaturisation des composants permet également d'imaginer l'apparition prochaine de stylos embarquant toujours plus de dispositifs et possédant de plus amples capacités de calcul pour les exploiter. Des dispositifs de localisation, type GPS, permettraient par exemple de connaitre la position de l'utilisateur et d'interpréter ses interactions en contexte. À plus long terme, on peut également envisager l'intégration de dispositifs de sortie avancés comme les pico-projecteurs [38]. Ainsi, les stylos numériques tendront vraisemblablement à devenir bien plus que de simples dispositifs d'entrée et de numérisation de tracés, ce qui laisse présager l'émergence de nombreux usages encore inexplorés.

\section{CONCLUSION}

Dans cet article, nous avons présenté un espace de caractérisation du stylo numérique. Après avoir proposé une définition de cet outil, nous avons décrit les axes de classification de notre espace en les illustrant par des exemples de l'état de l'art (qui figurent également dans une table synthétique [45] fournie en annexe). Cet espace permet la comparaison des techniques existantes et a pour but d'aider au développement d'applications exploitant cette technologie. Nous nous appuierons sur cet espace et poursuivrons son développement au cours de nos travaux futurs, lesquels s'effectueront dans le cadre de la classe numérique.

\section{BIBLIOGRAPHIE}

1. Arthur, A. M., Lunsford, R., Wesson, M., and Oviatt, S. (2006). Prototyping novel collaborative multimodal systems: simulation, data collection and analysis tools for the next decade. International Conference on Multimodal interfaces. ACM ICMI '06, pp. 209-216.

2. Barthelmess, P., Kaiser, E., Huang, X., McGee, D., and Cohen, P. (2006). Collaborative multimodal photo annotation over digital paper. International Conference on Multimodal interfaces. ACM ICMI '06, pp. 4-11.

3. Bernstein, M., Robinson-Mosher, A., Yeh, R. B., R. Klemmer, S. (2006). Diamond's Edge: From Notebook to Table and Back Again. (Poster). ACM UbiComp '06.

4. Brandl, P., Forlines, C., Wigdor, D., Haller, M., Shen, C. (2008). Combining and Measuring the Benefits of Bimanual Pen and Direct-Touch Interaction on Horizontal Interfaces. In Proceedings of AVI 08, pp. 154-161.

5. Brandl, P., Haller, M., Hurnaus, M., Lugmayr, V., Oberngruber, J., Oster, C., Schafleitner, C., and Billinghurst, M. (2007). An Adaptable Rear-Projection Screen Using Digital Pens And Hand Gestures. In International Conference on Artificial Reality and Telexistence. IEEE. ICAT '07, pp. 49-54.
6. Brandl, P., Haller, M., Oberngruber, J., Schafleitner, C. (2008). Bridging the Gap Between Real Printouts and Digital Whiteboard. In Proceedings of AVI 08, pp. 31-38.

7. Cao, X. and Balakrishnan, R. (2006). Interacting with dynamically defined information spaces using a handheld projector and a pen. User interface Software and Technology. ACM UIST '06, pp. 225-234.

8. Costa-Cunha, P. and Mackay, W. (2003). Augmented paper and Anoto stylus. Conference Francophone Sur L'interaction Homme-Machine. ACM IHM '03, pp. 232-235.

9. Decurtins, C., Norrie, M. C., and Signer, B. (2003). Digital annotation of printed documents. Conference on Information and Knowledge Management. ACM CIKM '03, pp. 552-555.

10. Ellis, C. A., Gibbs, S. J., and Rein, G. (1991). Groupware: some issues and experiences. Commun. ACM 34, 1 (Jan. 1991), 39-58.

11. Fekete, J. and Beaudouin-Lafon, M. (1996). Using the multi-layer model for building interactive graphical applications. User interface Software and Technology. ACM UIST '96, pp. 109-118.

12. Guimbretière, F. (2003). Paper augmented digital documents. User interface Software and Technology. ACM UIST '03, pp. 51-60.

13. Hurlbutt, T. and Klemmer, S. R. (2006). Bridging the gap: fluidly connecting paper notecards with digital representations for story/task-based planning. ACM CHI '06, pp. 887-892.

14. Jiang, H., Yeh, R. B., Winograd, T., Shi, Y., DigiPost: Writing on Post-its with Digital Pen to Support Collaborative Editing Tasks on Tabletop Displays. (Poster), UIST'07.

15. Konishi, K. and Ikeda, N. F. (2007). Data model and architecture of a paper-digital document management system. Symposium on Document Engineering . ACM DocEng '07, pp. 29-31.

16. Lecolinet, E. and Mouret, G. (2005). TACTIBALL,TACTIPEN,TACTITAB Ou comment « toucher du doigt » les données de son ordinateur. Conference Francophone Sur L'interaction HommeMachine. ACM IHM '05, pp. 227-230.

17. Lee, J. C., Dietz, P. H., Leigh, D., Yerazunis, W. S., and Hudson, S. E. (2004). Haptic pen: a tactile feedback stylus for touch screens. ACM UIST '04, pp.291-294.

18. Leithinger D., Haller M. (2007). Improving Menu Interaction for Cluttered Tabletop Setups with UserDrawn Path Menus Proceedings of IEEE Tabletop 2007, pp. 121-128. 
19. Liao, C., Guimbretière, F., and Hinckley, K. (2005). PapierCraft: a command system for interactive paper. User interface Software and Technology. ACM UIST '05, pp. 241-244.

20. Liao, C., Guimbretière, F., and Loeckenhoff, C. E. (2006). Pen-top feedback for paper-based interfaces. User interface Software and Technology . ACM UIST '06, pp. 201-210.

21. Mackay, W. E., Pothier, G., Letondal, C., Bøegh, K., and Sørensen, H. E. (2002). The missing link: augmenting biology laboratory notebooks. User interface Software and Technology. ACM UIST '02, pp. 41-50.

22. Nigay, L., Coutaz, J., Espaces Conceptuels pour l'Interaction Multimédia et Multimodale. TSI, spéciale Multimédia et Collecticiel, AFCET \& HERMES, 1996.

23. Norman, D. A. and Draper, S. W. (1986). User Centered System Design; New Perspectives on HumanComputer Interaction. Lawrence Erlbaum Associates, Inc.

24. Norrie, M. C., Signer, B., and Weibel, N. (2006). Print-n-link: weaving the paper web. Document Engineering. ACM DocEng '06, pp. 34-43.

25. Pintaric, T., Kauffman, H. Affordable InfraredOptical Pose-Tracking for Virtual and Augmented Reality. IEEE VR Workshop on Trends and Issues in Tracking for Virtual Environments, Shaker Verlag, pp. $44-51$.

26. Sellen, A. J. and Harper, R. H. (2003) The Myth of the Paperless Office. MIT Press.

27. Signer, B. and Norrie, M. C. (2007). PaperPoint: a paper-based presentation and interactive paper prototyping tool. Conference on Tangible and Embedded interaction. ACM TEI '07, pp. 57-64.

28. Signer, B., Norrie, M. C., Grossniklaus, M., Belotti, R., Decurtins, C., and Weibel, N. (2006). Paperbased mobile access to databases. Conference on Management of Data. ACM SIGMOD '06, pp. 763765.

29. Song, H., Guimbretière, F., Hu, C., and Lipson, H. (2006). ModelCraft: capturing freehand annotations and edits on physical 3D models. ACM UIST '06, pp. 13-22.

30. Weibel, N., Norrie, M. C., and Signer, B. (2007). A model for mapping between printed and digital document instances. Document Engineering. ACM DocEng '07, pp. 19-28.

31. Weibel, N., Signer, B., Ponti, P., Norrie, M. C. (2007). PaperProof: A Paper-Digital Proof-Editing System. CoPADD'07.
32. Wellner, P. 1993. Interacting with paper on the DigitalDesk. Commun. ACM 36, 7 (Jul. 1993), 8796.

33. West, D., Quigley, A., and Kay, J. (2007). MEMENTO: a digital-physical scrapbook for memory sharing. Personal Ubiquitous Comput.pp. 313-328.

34. Yeh, R. B., Brandt, J., Boli, J., R. Klemmer, S. (2006). Interactive Gigapixel Prints: Large, PaperBased Interfaces for Visual Context and Collaboration. ACM Ubicomp ,06.

35. Yeh, R., Liao, C., Klemmer, S., Guimbretière, F., Lee, B., Kakaradov, B., Stamberger, J., and Paepcke, A. (2006). ButterflyNet: a mobile capture and access system for field biology research. SIGCHI Conference on Human Factors in Computing Systems. ACM CHI '06, pp. 571-580.

36. Anoto Technology Site Web. http://www.anoto.com/

37. Article sur le stylo par Wikipedia : http://fr.wikipedia.org/wiki/Stylo/

38. Article sur les pico-projecteurs par Wikipedia: http://fr.wikipedia.org/wiki/Picoprojecteur

39. Epos Technology site Web. Disponible à l'adresse http://www .epos-ps.com/.

40. Leapfrog Fly Fusion Pentop Computer Site Web. http://www.flyworld.com/

41. Leapfrog Fly Pentop Computer Site Web. http://www.flypentop.com/view/page.home/home

42. Livescribe Pulse Smartpen Site Web. http://www.livescribe.com

43. Oxford Easybook Site Web. Disponible à l'adresse http://www .oxfordeasybook.com/

44. Scrivo.1 Site Web. Disponible à l'adresse https://www.plawa.com/fr/plawa/articles/digitations/ mobilities1/scrivo1/

45. Table synthétique de l'espace de caractérisation: http://www.malacria.fr/ihm08/digitalpen.html 